\title{
A Comparative Study of Network Performance between ContikiMAC and XMAC Protocols in Data Collection Application with ContikiRPL
}

\author{
Vu Chien Thang \\ Faculty of Electronics and Communications Technology, University of Information and \\ Communications Technology, Thai Nguyen, 250000, Viet Nam \\ E-mail: vcthang@ictu.edu.vn
}

Received: 03 May 2019; Accepted: 18 July 2019; Published: 08 August 2019

\begin{abstract}
This paper will present several research results evaluating the performance of ContikiMAC and XMAC protocols in data collection application with the RPL routing protocol. Simulation results show that ContikiMAC protocol gets better efficiency compared with XMAC protocol in both successful data delivery ratio and average energy consumption in the network. ContikiMAC protocol also performs well in high-density network condition. Meanwhile, successful data delivery ratio of XMAC protocol significantly reduced when the network density increases. The evaluating simulation results in this paper are an important basis for scientists to continue developing applications for wireless sensor networks in the future.
\end{abstract}

Index Terms-ContikiMAC protocol, XMAC protocol, wireless sensor network, energy-efficient MAC protocol, network performance evaluation.

\section{INTRODUCTION}

Wireless Sensor Network (WSN) is an infrastructure including sensing (measuring), processing and communicating components in order to provide administrators the ability to measure, observe and impact again with events, phenomena in a defined environment. Typical applications of wireless sensor networks include data collection, military, monitoring, and medicine, etc.

Wireless sensor nodes often use limited power (usually using battery), have a long operating time (from several months to several years). Most wireless sensor nodes are equipped with low-power radio transceivers. These radio transceivers are one of the most energy-consuming components. To conserve energy, radio transceivers need to be turned off. When radio transceivers are turned off, they cannot listen to transmissions from other nodes.

Not listening on the radio transceiver has affected the construction of the network topology for wireless sensor networks. In that state, only the star topology is appropriate to wireless sensor networks. In a star topology, the central node (sink node) has its radio turned on all the time. This node is supplied with external power.
All other nodes are powered by batteries and keep their radio transceivers turned off to save energy. Only when sending data, they will turn on the radio transceivers. The only node that they can transmit to is the central node because all other nodes have turned off the radio transceivers. In some narrow-scale applications, star topology is proper.

In order to expand the range of the network, nodes must have the ability to communicate with each other. In that state, the topology can provide redundant paths through the network, which increases reliability for the network. If a node runs out of power, the network can reroute flow around the faulty node. This is the mesh topology. In order to form the mesh topology, the radio transceivers of the nodes need to be controlled to turn off when they are not in use but must be turned on when a neighboring node wants to communicate. Therefore, a general protocol is needed so that nodes can communicate with each other.

In this paper, ContikiMAC and XMAC protocols are studied and evaluated in data collection application with the RPL routing protocol. Some evaluation metrics used such as the data delivery ratio, average power consumption, average number of parent node changes, and average hop count in the network. The performance of the network will be simulated and evaluated when the network density changes. These simulation results are useful for scientists to develop applications with different network density such as smart water [1], smart grid, smart agriculture, smart environment, etc.

\section{RELATED WORKS}

In the past, there have been a number of proposed protocols for wireless sensor networks [2, 3]. Initial studies have shown that energy was significantly saved in comparison with the cases where radio transceivers are often turned on. Some protocols such as S-MAC [4] reduced the average turned-on time of the radio from $100 \%$ to $35 \%$. WiseMAC protocol [5] reduced to even $20 \%$. 


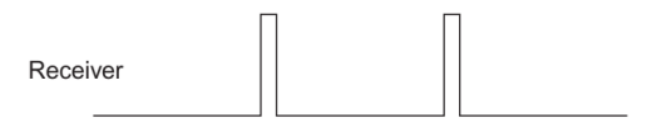

Fig.1. The radio transceivers are turned on/off periodically

One of the simplest energy-saving protocols is the LPL protocol [2]. This protocol achieves low power operation by turning off the radio in most of the time and periodically turning it on for a short period. By keeping the radio turned on for a short period of time, such protocol allows the sensor node to absolutely receive transmissions from neighboring nodes. This process is illustrated in figure 1.

To send a packet to a node, the sender first sends a train of short packets called strobes. When the receiver listens to a strobe, the receiver will turn on its radio transceiver to wait for data packets. The strobe train must be long enough for all neighboring nodes to listen at least once with in the period. This is shown in figure 2 .

Sender 图 图 图 图 图 圆 圆 图 图 图 $\square$

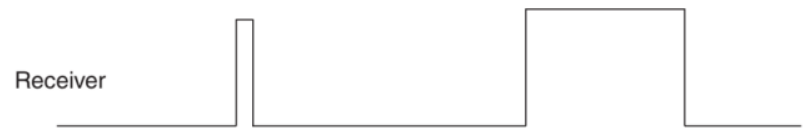

Fig.2. Operation of LPL protocol

However, it can be seen that, through analysis, the LPL protocol still has some disadvantages. First, the strobes wake up every node, not only the one receiving the final packet. This wastes energy for all other neighboring nodes because they have to turn on the radio to receive packets that are not sent to them. Second, the transmission of each packet will take a long time because if the receiving node turns off the radio for a second, the strobe train must be sent during a second. This also causes energy loss for the sending node.

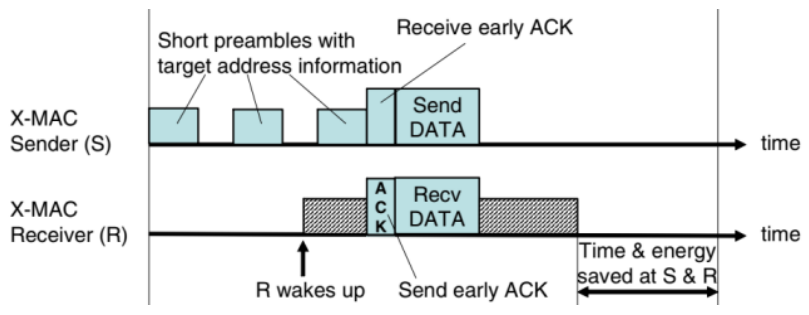

Fig.3. Operation of XMAC protocol [6]

In the paper [6], the authors proposed the XMAC protocol. XMAC protocol reaches higher energy efficiency than the LPL protocol. Before sending a data packet, the sender will send a train of short preambles. The short preamble carries address information of the destination node. When neighboring nodes receive a short preamble, it will check the information about destination node's address. If the address of destination node matches with that of receiving node, it will send a confirmation message ACK to the sender while still enabling the radio to wait for receiving the data packet. After receiving the
ACK message, the sender will send the data packet to the receiver. Conversely, if the address of destination node does not match with the address of the receiving node, the receiving node will turn off its radio. Thus, the XMAC protocol is more optimized than the LPL protocol because the waiting time for receiving data packets is shortened and nodes which are not the destination will quickly switch to sleep mode to save energy. Figure 3 illustrates the operation of the XMAC protocol.

ContikiMAC protocol is proposed in the paper [7]. Figure 4 depicts the operation of this protocol.

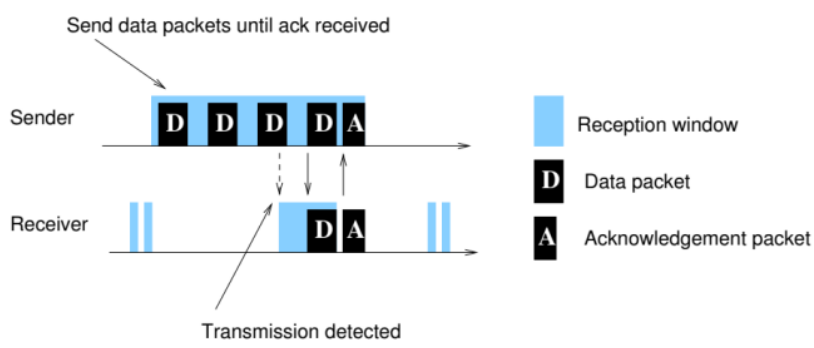

Fig.4. Operation of ContikiMAC protocol [7]

In order to send a data packet, the sender repetitively sends the same packet until a confirmation message is received. The nodes in the network turn off the radio for most of the time and periodically turn on to check the transmission channel. If a data packet is detected on the transmission channel, the receiver will always turn on the radio to receive data packets. The receiving node will check the data packet, if the data packet is sent to it, it will confirm to the sender with an ACK message. As such, ContikiMAC protocol is designed to be simple, easily implement and does not need to use signaling messages as well as additional headers.

\section{CONNECTIVITY MODEL OF WIRELESS SENSOR NETWORK}

This paper will focus on evaluating the XMAC and ContikiMAC protocols in data collection application with the RPL protocol [8]. The RPL protocol is a protocol designed for Low-Power and Lossy Networks (LLNs) with limited resource nodes and interconnected by lossy links (loss packet). The RPL protocol is a distance vector protocol. This protocol builds a topology consisting of one or more Destination Oriented Direct Acyclic Graph (DODAG). The route is constructed from nodes in the network to one of the root nodes of the DODAG [9].

Figure 5 shows the implementation of RPL protocol in the uIPv6 communication stack of the Contiki operating system [11]. The uIPv6 calls the ContikiRPL module when receiving ICMPv6 messages and discovering neighbors. ContikiRPL module calls the uIPv6 stack to install routes to the IPv6 routing tables.

Figure 6 demonstrates the network topology model which is considered in this paper. The network is divided into many different small clusters. Since the clusters are the same, only one cluster is simulated and evaluated. 


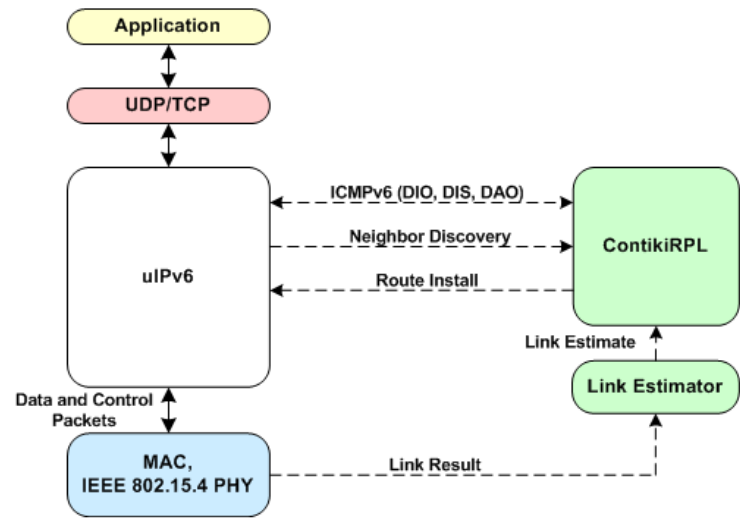

Fig.5. Implementation of the RPL protocol in ContikiOS [10]

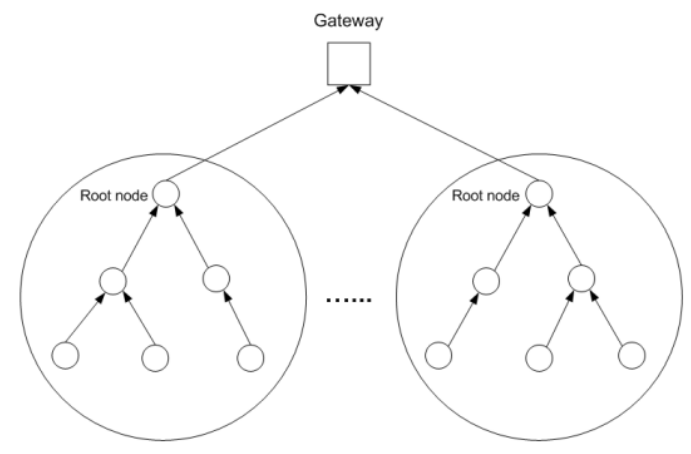

Fig.6. The network topology is divided into many different small clusters

The assumptions set for the simulation problem are: nodes are heterogeneous and there are two types of nodes, sensor nodes, and root nodes; The sensor nodes read and send data to the root nodes via other intermediate sensor nodes. Root nodes collect data and directly send data to a gateway; During the entire operation of the network, nodes transmit in the constant power level. No data aggregation is made in the network. All data collected by the root nodes are sent to the gateway; The sensor nodes are fixed, the network is considered static.

The connectivity of a wireless sensor network is described by a graph $\mathrm{G}=(\mathrm{V}, \mathrm{E})$, where $\mathrm{V}$ (vertices) is the set of sensor nodes, and $E$ (edges) describes the adjacency relation between nodes. That is, for two devices $\mathrm{u}, \mathrm{v} \in \mathrm{V},(\mathrm{u}, \mathrm{v}) \in \mathrm{E}$ if $\mathrm{v}$ is adjacent to $\mathrm{u}$. In an undirected graph, it holds that if (u,v) $\in \mathrm{E}$, then also (v, $\mathrm{u}) \in \mathrm{E}$; that is, edges can be represented by sets $\{\mathrm{u}, \mathrm{v}\}$ $\in \mathrm{E}$ rather than tuples. The classic connectivity model is the so-called unit disk graph (UDG). The UDG model is idealistic: In reality, radios are not omnidirectional, and even small obstacles such as plants can change connectivity.

In wireless networks, the communication medium is shared and transmissions are exposed to interference. Concretely, a node $\mathrm{u}$ may not be able to correctly receive a message of an adjacent node $\mathrm{v}$ because there is a concurrent transmission nearby. In some sense, an interference model explains how concurrent transmissions block each other.

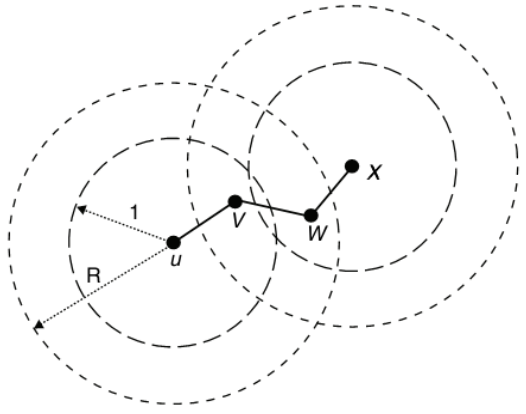

Fig.7. The UDI model [12]

In this paper, the UDI model is used for simulation [12]. Nodes are situated arbitrarily in the plane. Two nodes can communicate directly if and only if their Euclidean distance is at most 1, and if the receiver is not disturbed by a third node with Euclidean distance less or equal a constant $\mathrm{R} \geq 1$.

Figure 7 describes the UDI model considered in this paper. The UDI model has two radii: a transmission radius (length 1 ) and an interference radius (length $R \geq 1$ ). In this figure, node $\mathrm{v}$ is not able to receive a transmission from node $u$ if node $x$ concurrently transmits data to node $\mathrm{w}$ - even though $\mathrm{v}$ is not adjacent to $\mathrm{x}$.

\section{PERformance EVAluation of ContikimaC AND XMAC PROTOCOLS IN DATA COLLECTION APPLICATION WITH CONTIKIRPL}

\section{A. Evaluation Metrics}

The performance of ContikiMAC and XMAC protocols will be evaluated and compared through some of the following evaluation metrics.

\section{Data Delivery Ratio:}

The first metric is the data delivery ratio (DDR). We define DDR as the ratio between the number of data packets received at the root and the total number of sent data packets by all nodes in the whole network.

$$
\operatorname{DDR}(\%)=\frac{N_{\text {received }}}{N_{\text {data }}} .100 \%
$$

In (1), $N_{\text {received }}$ is the number of data packets received at the root; $N_{\text {data }}$ is the number of data packets sent by all nodes in the network. The higher the DDR is, the better the communication efficiency of the network becomes. Clearly, DDR equals to 1 indicates that the network can deliver all the data to the root node.

\section{Average energy consumption:}

In this paper, IRPL and RPL protocols have been evaluated based on the simulations. Tmote Sky hardware platform built on Cooja simulation tool was used [13]. To estimate the energy consumption of the Tmote Sky hardware platform, the software-based online energy estimation was used. The total energy consumption of the node is defined as [14]: 


$$
E_{\text {consumption }}=U\left(I_{a} t_{a}+I_{l} t_{l}+I_{t} t_{t}+I_{r} t_{r}+\sum_{i} I_{c i} t_{c i}\right)
$$

Where $U$ is the supply voltage, $I_{a}$ is the consumption current of the microcontroller when running, $t_{a}$ is the time in which the microcontroller has been running, $I_{l}$ and $t_{l}$ are the consumption current and the time of microcontroller in low power mode, $I_{t}$ and $t_{t}$ are the consumption current and the time of the communication device in transmit mode, $I_{r}$ and $t_{r}$ are the consumption current and the time of communication device in receive mode, $I_{c i}$ and $t_{c i}$ are the consumption and the time of other components such as sensors and LEDs...

Table 1. Energy Model of Tmote Sky.

\begin{tabular}{|c|c|c|}
\hline Component & State & Current \\
\hline \multirow{2}{*}{ MSP430 F1611 } & Active & $1,95 \mathrm{~mA}$ \\
\cline { 2 - 3 } & Low power mode & $0,0026 \mathrm{~mA}$ \\
\hline \multirow{2}{*}{ CC2420 } & Transmit $(0 \mathrm{dBm})$ & $17,4 \mathrm{~mA}$ \\
\cline { 2 - 3 } & Listen & $19.7 \mathrm{~mA}$ \\
\hline
\end{tabular}

Table 1 shows the energy model of Tmote Sky, where the consumption currents are from chip manufacturer data sheets [15]. In the energy model of Tmote Sky, the author only considers the main energy consumptions that are the radio transceiver, the microcontroller, and other small energy consumptions ignored.

\section{Average number of times to change parent node:}

The average number of parent node change is determined based on the statistics of the number of parent node changes for each node. Wireless sensor networks operate on lossy radio links. The radio links are often unstable quality and change frequently over time. Therefore, network topology also needs changing in order to adapt to the radio communication environment. To evaluate this adaptive change, the author relies on statistics of the average number of parent node changes in the whole network.

\section{Average hop count in the network:}

The hop count refers to the number of intermediate nodes through which data must pass between source and destination. Hop count is a rough measure of distance between two nodes.

\section{B. The Scenario of Evaluation}

With the assumptions set out in section 3 of this paper, a cluster model consists of sensor nodes randomly distributed in a grid area of $100 \mathrm{mx} 100 \mathrm{~m}$. Nodes periodically send data packets to the root node located at the center of the cluster. Figure 8 illustrates a cluster model including 35 nodes, the root node is No. 35 .

The parameters used over the time of evaluating simulation are summarized in Table 2. Radio communication model used in the simulation is the UDI model, in which the effective transmission range is 30 meters and the interference range is 50 meters. The network layer protocol used in the simulation scenario is the RPL protocol. The MAC layer protocol configured as
ContikiMAC and XMAC, respectively. Under normal operating conditions, each sensor node would send data packets to the root node with a random frequency of 1 packet/1 minutes.

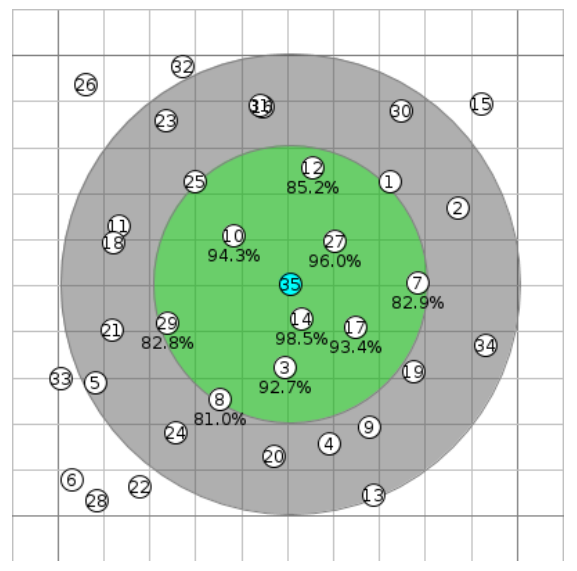

Fig.8. A simulated cluster model of 35 nodes

Table 2. Evaluating Simulation Scenario

\begin{tabular}{|c|c|}
\hline Parameters & Values \\
\hline Radio model & $\begin{array}{c}\text { UDI (Unit Disk Graph with } \\
\text { Distance Interference) }\end{array}$ \\
\hline Number of nodes & $25,30,35,40,45$ \\
\hline Network size (m x m) & $100 \times 100$ \\
\hline $\begin{array}{c}\text { Communication range of } \\
\text { node }\end{array}$ & $\begin{array}{c}\text { Transmission range: } 30 \mathrm{~m}, \\
\text { Interference range: } 50 \mathrm{~m}\end{array}$ \\
\hline Routing protocol & RPL \\
\hline Data packet interval & All nodes except the root node \\
\hline Data packet initialization & ContikiMAC, XMAC \\
\hline MAC protocol & \\
\hline
\end{tabular}

\section{Results of Simulation}

Post-simulation data is extracted, analyzed and graphed to make the comparison. Figures 9, 10, 11 and 12 below correspond to the simulation results comparing between the ContikiMAC protocol and XMAC protocol in terms of the performance of data delivery ratio, average power consumption, average number of parent node changes, and average hop count in the network.

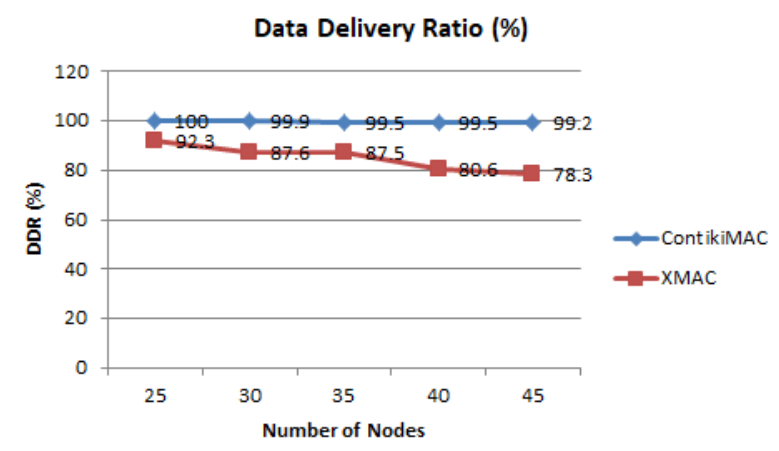

Fig.9. Comparison in terms of data delivery ratio

The simulation results in figure 9 show that the network operated by ContikiMAC protocol achieves efficiency in terms of data delivery ratio higher than that operated by the XMAC protocol. As the density of nodes 
in the network increases, the XMAC protocol presents a significant decline in the data delivery ratio (from $92.3 \%$ to $78.3 \%$ ). The XMAC protocol using a train of short preambles to synchronize the transmission time between the sender and receiver. Therefore, when the density of nodes in the network increases, the number of short preambles sent and received in the network also increases. This causes the conflict and loss of packets in the network. However, for ContikiMAC protocol, when the density of nodes in the network increases, the efficiency in terms of data delivery ratio in the network decreases insignificantly (from $100 \%$ to $99.2 \%$ ).

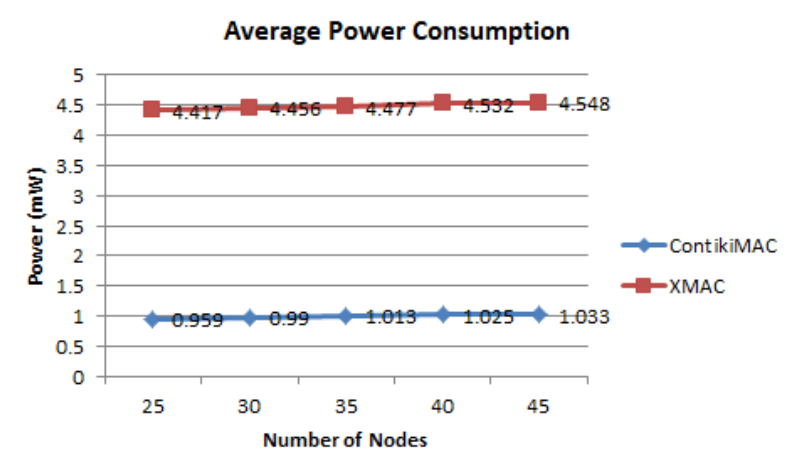

Fig.10. Comparison in terms of average power consumption in the network

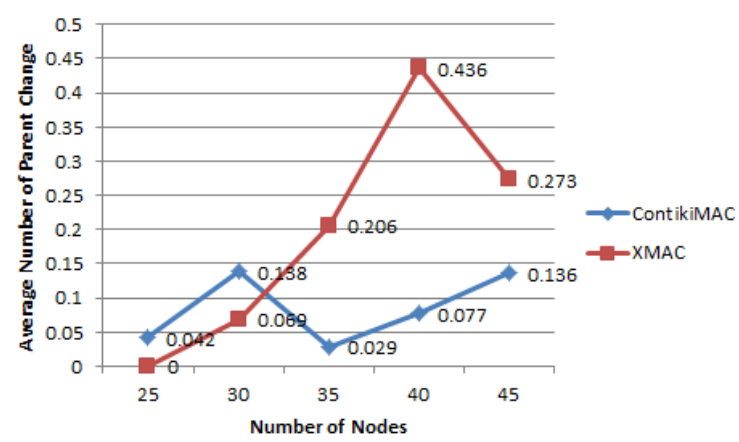

Fig.11. Comparison in terms of average number of parent node changes

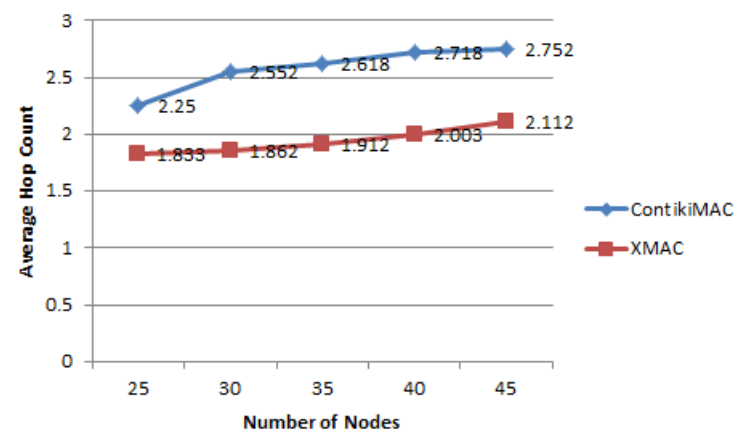

Fig.12. Comparison in terms of average hop count

Figure 10 illustrates that the network operated by ContikiMAC protocol gets better energy efficiency than that operated by the XMAC protocol. Compared to the XMAC protocol, the ContikiMAC protocol does not make any additional energy costs due to not sending short preambles.

In comparison of network stability (see figure 11), is can be seen that the network operated by ContikiMAC protocol reaches better stability than that operated by XMAC protocol. Based on simulation results, for ContikiMAC protocol, the average number of parent node changes in the network does not change much when the network density increases. However, for the XMAC protocol, the average number of parent node changes in the network increases significantly as the network density increases. As the network density increases, noise and conflict during transmission/reception also increase, thereby the data delivery ratio reduces and nodes tend to update the parent node to find out alternative routes. This makes the topology change. Figure 11 also shows that for low density networks, the topology is not changed much when the network operates under both the XMAC protocol and ContikiMAC protocol.

Figure 12 shows that the network operated by XMAC protocol has a lower average hop count than that operated by ContikiMAC protocol. Therefore, the number of hops that data packets need to be forwarded in the network under the XMAC protocol is lower than that of ContikiMAC protocol. The average hop count is related to the communication delay in the network.

\section{CONCLUSIONS}

This paper presented some evaluating results that compare the performance of ContikiMAC protocol and XMAC protocol in data collection application with the RPL routing protocol. The results of evaluating simulation show that the XMAC protocol works relatively well in low-density network condition. However, when the network density increases, the network's performance is significantly reduced. In all simulation scenarios, the ContikiMAC protocol always achieves good energy efficiency and better data delivery ratio than that of the XMAC protocol.

\section{REFERENCES}

[1] Vu Chien Thang, "A Solution for Water Factories in Vietnam using Automatic Meter Reading Technology," International Journal of Computer Network and Information Security (IJCNIS), Vol.10, No.8, pp.44-50, 2018.

[2] Jean-Philippe Vasseur, Adam Dunkels, "Interconnecting Smart Object with IP: The Next Internet," Morgan Kaufmann Publishers, 2010.

[3] Areeg Fahad Rasheed, A E Abdelkareem, "Performance Evaluation of MAC Protocols with Multi-Sink for Mobile UWSNs," International Journal of Computer Network and Information Security (IJCNIS), Vol.11, No.7, pp.1-7, 2019.

[4] Ye W, Silva F, Heidemann J., "Ultra-low duty cycle MAC with scheduled channel polling," In Proceedings of the 4th International Conference on Embedded Networked Sensor Systems. New York, NY: ACM Press; pp.321 334, 2006.

[5] El-Hoiydi A, Decotignie JD, Enz CC, Le Roux E. "WiseMAC, an ultra low power MAC protocol for the WiseNet wireless sensor network," In: SenSys, pp. $302-$ 303, 2003.

[6] M. Buettner, G. V. Yee, E. Anderson, and R. Han., "X- 
MAC: a short preamble mac protocol for duty-cycled wireless sensor networks," In Proceedings of 2nd ACM conference on Embedded Networked Sensor Systems (SenSys'06), pp. 307-320, 2006.

[7] A. Dunkels, "The ContikiMAC Radio Duty Cycling Protocol," SICS technical report, December 2011.

[8] Vasseur, J.P., Navneet Agarwal, Jonathan Hui, Zach Shelby, Paul Bertrand, Cedric Chauvenet, "RPL: the IP routing protocol designed for low power and lossy networks," In: Internet Protocol for Smart Objects (IPSO) Alliance, 2011.

[9] Vu Chien Thang, Nguyen Van Tao, "A Performance Evaluation of Improved IPv6 Routing Protocol for Wireless Sensor Networks," International Journal of Intelligent Systems and Applications, pp.18-25, 2016.

[10] N. Tsiftes, J. Eriksson, and A. Dunkels, "Low-Power Wireless IPv6 Routing with ContikiRPL," in Proceedings of the International Conference on Information Processing in Sensor Networks (ACM/IEEE IPSN), Stockholm, Sweden, 2010.

[11] A. Dunkels, B. Grönvall, and T. Voigt, "Contiki - a lightweight and flexible operating system for tiny networked sensors," in Proc. EmNets, 2004.

[12] Azzedine Boukerche, "Algorithms and Protocols for Wireless Sensor Networks," John Wiley \& Sons Inc., ISBN: 9780470396360, 2008.

[13] Fredrik Österlind, Adam Dunkels, Joakim Eriksson, Niclas Finne, and Thiemo Voigt, "Cross-level sensor network simulation with cooja," In Proceedings of the First IEEE International Workshop on Practical Issues in Building Sensor Network Applications, Tampa, Florida, USA, 2006.

[14] Adam Dunkels, Fredrik Osterlind, Nicolas Tsiftes, Zhitao He, "Software-based Online Energy Estimation for Sensor Nodes," Proceedings of the 4th workshop on Embedded networked sensors, 2007.

[15] https://insense.cs.st-andrews.ac.uk/files/2013/04/tmotesky-datasheet.pdf.

\section{Authors' Profiles}

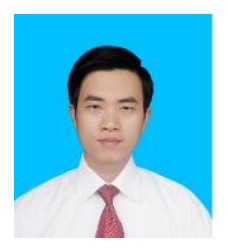

Vu Chien Thang received the MSc degree in Electronics and Communication Technology in 2009 from Hanoi University of Science and Technology and Ph.D. in Telecommunication Engineering in 2015 from Vietnam Research Institute of Electronics, Informatics, and Automation. He is currently a lecturer at Thai Nguyen University of Information and Communication Technology. His research interests include wireless sensor networks, internet of things, embedded systems.

How to cite this paper: Vu Chien Thang, "A Comparative Study of Network Performance between ContikiMAC and XMAC Protocols in Data Collection Application with ContikiRPL", International Journal of Computer Network and Information Security(IJCNIS), Vol.11, No.8, pp.32-37, 2019.DOI: $10.5815 / \mathrm{ijcnis.2019.08.04}$ 